\title{
Population Genetic Structure of Neoschoengastia Gallinarum in China Based on Mitochondrial DNA Markers
}

\section{Jia-Meng Tao}

South China Agricultural University

Saeed-El Ashram

Kafrelsheikh University

\section{Yuan Zhang}

South China Agricultural University

\section{Ya-Biao Weng}

South China Agricultural University

Rui-Qing Lin ( $\nabla$ rqlin@scau.edu.cn )

South China Agricultural University

\section{Research}

Keywords: Neoschoengastia gallinarum, Population genetic structure, Mitochondrial genome, China

Posted Date: December 29th, 2021

DOI: https://doi.org/10.21203/rs.3.rs-1186846/v1

License: (9) This work is licensed under a Creative Commons Attribution 4.0 International License. Read Full License 


\section{Abstract}

Background: Neoschoengastia gallinarum is a widespread agricultural pest in China.The larvae of $N$. gallinarum are parasitic on the body surface of poultry. Performance, carcass quality, and normal marketing of mite infected broilers are severely affected by pruritus and pockmark lesions on the body surface. In China, N. gallinarum research has primarily focused on occurrence regularity, biology, and control methods. The genetic structure, variation, and genetic relationship between the $N$. gallinarum populations in China are still unclear.

Methods: Genetic variations and structure among populations of $N$. gallinarum was examined and analyzed based on the nucleotide sequences of a $1522 \mathrm{nt}$ variable region of the mitochondrial tandem genes (COI, COII, and ND5) among 4 populations from 7 collection sites in southern China.

Results: A total of 192 individuals in 4 populations were analyzed. The tandem genes sequences were aligned, and 75 haplotypes were detected, 4 of these shared between populations. The range of haplotype diversity was from 0.860 (FJ) to 0.978 (GX). The pairwise $F_{\mathrm{ST}}$ values among populations were higher (0.096-0.551). The haplotype network mediation map and phylogenetic tree showed that the haplotypes were divided into two clade, Which did not completely follow the distribution rule of geographical populations. The AMOVA result showed that the percentage of variation within populations (72.94\%) was higher than that among populations (27.06\%). Neutral test and mismatch analysisrevealed that $N$. gallinarum had not experienced an obvious population expansion in recent historical periods, and the population size was relatively stable.

Conclusions: The $N$. gallinarum population showed high genetic diversity based on mitochondrial tandem genes analysis and strong ecological adaptability. Despite the fact that geogrphic isolation causes certain genetic differentiation among populations, $N$. gallinarum high gene flow among populations as a result of human trade activities, and there was no obvious geographical genetic structure.

\section{Introduction}

Neoschoengastia gallinarum (Trombiculidae) is a widespread agricultural pest in China. This mite was discovered and named by Hatori in Taiwan in 1920 [1], and is distributed in Malaysia, India, and other countries, with the highest annual incidence rate in southern China [2]. The larvae of $N$. gallinarum are parasitic on the body surface of poultry (especially free-range broilers), mainly on the inner side of the wings, chest, and leg skin of broilers. The feathers on the affected part fall off, and a volcanic ulcer lesion can be formed on the body surface, with varying numbers of worms visible in the center of the lesion. Performance, carcass quality, and normal marketing of mite-infected broilers are severely affected by pruritus and pockmark lesions on the body surface $[2,3]$.

In China, N. gallinarum research has primarily focused on occurrence regularity, biology, and control methods. The genetic structure, variation, and genetic relationship between the $N$. gallinarum populations in China are still unclear. Through the amplification and comparison of the ribosomal ITS2 sequence, the 
partial 18S rDNA sequence, the partial 28S rDNA sequence, and the partial mitochondrial cytochrome $c$ oxidase submit 1 ( $\mathrm{CO} /$ ) sequence of $10 \mathrm{~N}$. gallinarum from two geographical regions, Yunfu, Guangdong province and Putian, Fujian province. Our research team concluded that the rDNA was relatively conservative, and ITS2 could be used as a molecular identification marker of $N$. gallinarum, which also greatly promoted the subsequent genetic research [4]. Genetic diversity and phylogenetic connections across species may be studied using mitochondrial DNA (mtDNA) sequencing data. This data can be utilized to precisely analyze gene flow and variations between populations $[5,6]$. Among them, $\mathrm{COI}$, cytochrome c oxidase submit 2 (COI), and NADH dehydrogenase subunit 5 (ND5) are ideal molecular markers for taxonomic and phylogenetic studies and have been successfully applied to molecular identification, germline development, and genetic variation of many animal parasites [7, 8]. This study used mtDNA tandem genes to investigate the genetic characteristics of $N$. gallinarum populations in southern China.

\section{Materials And Methods}

\section{Mite collection and morphological identification}

Between July 2019 and May 2020, 192 N. gallinarum were collected from yellow-feathered chickens in seven different locations across southern China (Fig.1). The mites were obtained from the skin with crateriform lesions using forceps. The chicken was set free immediately after completing the mite examination. Individual mite specimens were stored at $-20^{\circ} \mathrm{C}$ in $1.5 \mathrm{ml}$ microcentrifuge tubes. They were then morphologically identified based on the taxonomic keys of Trombiculidae. All specimens identified as trombiculid mites were selected and further used for molecular identification to confirm the species identification. The $N$. gallinarum identity was diagnosed by a PCR assay based on ITS2 rDNA sequences [4] .

\section{Genomic DNA isolation and sequencing of the COI, COII, and ND5 mitochondrial gene fragments}

The genomic DNA of a single mite was isolated using the Genomic Mini Kit (TIANamp Genomic DNA, Beijing, China), following the manufacturer's protocol. The partial mitochondrial genes COI, COII, and ND5 were used as genetic markers for amplification. The primers were designed based on the published universal primers for arthropod mtDNA [9]. After the corresponding gene sequences were amplified, the specific amplification primers for gene fragment intervals were designed using Primer Premier 5.0. The PCR amplifications were performed in a final volume of $25 \mu$ l containing genomic DNA, Premix Ex Taq (Takara Bio Inc., China), and $10 \mu \mathrm{mol}$ of forward and reverse primers for each genetic marker. PCR was performed with an initial denaturation at $94^{\circ} \mathrm{C}$ for 5 min, followed by 35 cycles of denaturation at $94{ }^{\circ} \mathrm{C}$ for $30 \mathrm{~s}$, annealing at $52{ }^{\circ} \mathrm{C}$ (for CO/primers) and $54{ }^{\circ} \mathrm{C}$ (for COll and ND5 primers) for $30 \mathrm{~s}$, extension at $72{ }^{\circ} \mathrm{C}$ for $40 \mathrm{~s}$, and a final extension at $72{ }^{\circ} \mathrm{C}$ for $7 \mathrm{~min}$. The PCR products were subsequently purified using the TaKaRa MiniBEST DNA Fragment Purification Kit Ver.4.0 (Takara Bio Inc., China). The purified PCR products were sequenced with the same primers as conventional PCR by Sanger sequencing using a 3730 analyzer (Thermo Scientific, USA). 


\section{Data analyses}

Multiple gene sequence alignments were performed and edited by MEGA 7.0 [10]. The gene sequences of COI, COII, and ND5 of each sample were connected into a single sequence by Phylosuite software [11], and the tandem gene sequence homogeneity test was performed using PAUP 4.0 Beta 10 software [12]. The inconsistent length difference test (ILD test) was used to determine whether the three genes could be analyzed jointly.

The nucleotide composition and mutation site of the tandem gene were calculated in MEGA 7.0 software. The haplotypes $(H)$, the haplotype diversity $\left(H_{d}\right)$, the average number of nucleotide differences $(K)$, the average number of mutations per sequence $(\theta)$, number of variable sites $(S)$, and nucleotide diversity $(P I)$ of different geographical population sequences were analyzed by DnaSP 6.0 [13], and Tajima's $D$ and Fu's Fs neutral tests were carried out to estimate the population expansion and the degree of genetic differentiation among different populations.

Mismatch distributions and hierarchical analysis of molecular variance (AMOVA) were calculated using ARLEQUIN 3.11 [14]. The pairwise $F_{\mathrm{ST}}$ values for the short-term genetic distance between populations were estimated with the method of Slatkin and tested for significance by permutation. The gene flow [Nm $\left.\left(1-F_{\mathrm{ST}}\right) / 4 F_{\mathrm{ST}}\right][15]$ between locations was estimated using pairwise $F_{\mathrm{ST}}$. MEGA 7.0 based on the $\mathrm{ML}$ model, Amblyomma Americanum (Acarina: Ixodidae, GenBank: DQ168131) was used as the outer group to construct the haplotype phylogenetic tree, and the haplotype intermediary network diagram was constructed based on the Median-Joining model using Popart 1.7 [16].

\section{Results}

\section{Sample collection and identification}

N. gallinarum samples were collected from 7 sites in 4 provinces in China. Four populations were analyzed, in which GD and GX consisted of specimens collected from two or three sites in proximity to each other (Table 1 and Fig. 1). There were 192 individuals of $N$. gallinarum, which were identified by PCR assay.

\section{Sequence characteristics of tandem gene}

A segment of mtDNA, corresponding to the coding region of COI, COII, and ND5, was successfully amplified from $N$. gallinarum. A sequence of $1522 \mathrm{nt}$ was obtained and analyzed. No insertion or deletion was detected across all samples. The conserved sites were 1363, the variable sites 159 . Of these, 10 were singletons and 149 were parsimony informative.

\section{Genetic diversity}

The haplotypes ranged from $4(\mathrm{FJ})$ to $31(\mathrm{GD})$, corresponding with the range of nucleotide diversity (PI) and diversity of the average number of nucleotide differences $(K)$, which were $0.00718 \pm 0.00217$ (mean \pm 
standard deviation, SD) (FJ) - 0.43886 \pm 0.00102 (GD) and 13.760 (FJ) - 1309 (GD), respectively (Table 2). The haplotype diversity $\left(\mathrm{H}_{d}\right)$ and nucleotide diversity of the population $(P I)$ were $0.965 \pm 0.014$ and $0.26069 \pm 0.00109$, respectively, which showed higher haplotype diversity and higher nucleotide diversity $(P \mathbb{\mathbb { }} 0.01)$, and the genetic diversity was relatively abundant.

A total of 75 haplotypes were detected in 192 (75/192; 39.06\%) of the $N$. gallinarum individuals. The range of haplotype diversity $\left(\mathrm{H}_{d}\right)$ was from $0.860 \pm 0.017$ (mean \pm standard deviation, $\mathrm{SD}$ ) (FJ) to $0.978 \pm 0.011(\mathrm{GX})$. Among the 75 haplotypes, 4 were shared haplotypes $(\mathrm{H} 1, \mathrm{H} 5, \mathrm{H} 38$, and $\mathrm{H} 41)$, and the remaining 71 were population-specific haplotypes. Among the four shared haplotypes $(\mathrm{H} 1, \mathrm{H} 5, \mathrm{H} 38$, and H41), H1 has the highest frequency (26/192), followed by H5 (14/192), and GD and FJ share. Meanwhile, it was found that two or more populations sharing haplotypes were GD and FJ, while the haplotypes of the GX and JX populations were both population-specific haplotypes. Among all haplotypes, 37 haplotypes only appeared once. Based on the tandem genes, the pairwise fixed index $F_{\mathrm{ST}}$ of different populations of $N$. gallinarum was calculated and analyzed by Arlequin 3.5.2.2, and the gene flow $\mathrm{Nm}$ among geographic populations was calculated using the gene flow formula $N m \approx\left(1-F_{\mathrm{ST}}\right) / 4 F_{\mathrm{ST}}$. The results showed that the $F_{\mathrm{ST}}$ value between GD and GX populations was less than 0.25 , and there was lower genetic differentiation, and gene exchange between populations was more frequent $(1<\mathrm{N} m<4)$. In addition, there was a high degree of genetic differentiation among other populations $\left(F_{\mathrm{ST}}>0.25\right)$, among which the genetic differentiation between FJ and JX populations was the highest $\left(F_{\mathrm{ST}}=0.55123\right)$ and the level of gene flow was the lowest $(\mathrm{N} m=0.20353)$ (Table 3).

Based on tandem gene sequences, molecular analysis of variance (AMOVA) was performed on the genetic variation among different populations and different individuals within all geographic populations, respectively (Table. 4) The results showed that the genetic variation within geographical populations accounted for $72.94 \%$ of the total variation, while the variation among geographical populations only accounted for $27.06 \%$, which indicated that the molecular variation of $N$. gallinarum mainly came from within the population, followed by geographical isolation.

\section{Evolution of haplotype system and historical population dynamics}

The haplotype-based ML phylogenetic tree was constructed using MEGA 7.0.0 and the haplotype network mediation map was constructed using PopART 1.7 to explain the relationship between the haplotypes.

The relationships among the $N$. gallinarum haplotypes were drawn using $\mathrm{MJ}$, visualizing the haplotype frequencies and mutational steps among the haplotypes (Fig. 2 ). Each haplotype is represented by a circle in which the circle size is proportional to the haplotype frequency. Mutations between haplotypes are indicated by lines representing mutations from the common haplotype. The haplotype network structure has a star-like phylogeny, with the most relevant single haplotypes surrounding the common haplotype. Phylogenetic tree results showed that the 75 haplotypes differentiated into two clades: clade $A$ and clade B (Fig. 3). Clade A included 44 haplotypes and clade B included 31 haplotypes. Although obvious aggregation occurred among different haplotypes, some haplotypes of the same geographical 
population gathered into a branch preferentially, and the populations sharing the same haplotype gathered together. The distribution of haplotypes in evolutionary branches did not completely follow the distribution law of geographical populations, and for $N$. gallinarum, no geographical populations with significant genetic differences were formed.

The results of the haplotype intermediate network diagram were consistent with those of the phylogenetic tree analysis, and no obvious geographical genetic structure was formed. The haplotypes in the diagram presented multiple star-like distributions and formed multiple groups, but the haplotypes of different populations were distributed in each group, which further verified the results of the molecular analysis of variance in Table 4, namely, that the genetic differentiation among populations was small, and the genetic differentiation was mainly from within the population.

Based on the tandem genes, the historical dynamics of the $N$. gallinarum population in chickens were analyzed by using the Tajima's $D$ and Fu's Fs tests (Table 2). The results showed that Tajima $D$ and Fu's Fs of each population were both positive, but the $P$ values were not significant $(P<0.05$ indicating significance), indicating that the population size of $N$. gallinarum was relatively stable in history.

The historical dynamics of the $N$. gallinarum population were assessed by calculating the difference in the nucleotide pair for the $N$. gallinarum population as a whole and each geographic population using DnaSP 6.0 (Fig.4). The pairwise difference diagrams of the $N$. gallinarum population as a whole and four geographical populations showed that the nucleotide pairwise difference curves showed obvious multimodal distribution, which was consistent with the results of the neutral test, further indicating that the $N$. gallinarum population had not experienced any significant population expansion events in the recent historical period, and the population size was relatively stable.

\section{Discussion}

In this study, we analyzed the genetic structure of the population using mtDNA gene sequences and revealed information about genetic diversity, genetic differentiation, gene flow, and selection within and between populations. This study identified significant genetic distances between different populations of N. gallinarum, which indicates significant genetic differences between the populations of N. gallinarum.

The results of various molecular marker studies revealed some differences, which were due to differences in gene sequence evolution speeds. For the $N$. gallinarum samples with the same base difference sites in the $\mathrm{CO} /$ gene sequence, the base difference sites in the COll gene and the ND5 gene were different, which might also be due to the difference in the evolutionary rates of gene sequences, in which $\mathrm{CO}$ and $\mathrm{COII}$ genes were relatively conservative, and the $N D 5$ gene had a rapid evolution rate [17-19], so a total of up to 75 haplotypes were detected based on the tandem gene of $192 \mathrm{~N}$. gallinarum samples. In addition, the length of molecular markers, the number of population samples, whether the population suffers from the bottleneck effect, and other factors all influence the level of genetic diversity [20]. To better understand the genetic diversity of $N$. gallinarum populations, more $N$. gallinarum populations must be studied, and longer gene sequences or combinations of multiple gene sequences must be studied. Geographically, the 
population of $N$. gallinarum is highly isolated, reducing genetic variation while increasing the number of related haplotypes. Haplotype sharing occurs between populations, even geographically distant populations. By matching and comparing the populations in different geographical distribution areas, the $F_{\mathrm{ST}}$ value is moderate, and the genetic variation within a population is far greater than that among the populations. This observation shows that there was a certain gene exchange among the populations.

Our analyses revealed strong genetic differentiation between JX and other geographic regions. Low gene flow between JX and other geographic regions further confirmed this result (Table 3). When the Nm value of gene flow between populations is less than one, it is generally assumed that the limited gene flow will promote genetic differentiation of the population [15]. These findings suggest that geographical isolation has an impact on the genetic differentiation of $N$. gallinarum. The larval stage of the $N$. gallinarum is only found on the surface of poultry, and the individual is small and wingless, with limited migration ability, limiting the $N$. gallinarum's ability to migrate. Therefore, there is little gene flow among geographical populations in the different provinces, and the degree of genetic differentiation is high. However, the $F_{\mathrm{ST}}$ values of GD and GX populations based on tandem gene molecular markers showed that there was no genetic differentiation between these two populations, $F_{\mathrm{ST}}$ was less than 0.05 , and the $N_{\mathrm{m}}$ value of gene flow was high (2.34794). There is no obvious genetic differentiation between GX and FJ populations, as well as GX and FJ populations. The above findings showed that geographic barriers are not the primary source of mite genetic variation, and that among the haplotypes defined by tandem gene sequences, there was a shared haplotype in four provinces, which could be due to ancestral haplotypes or certain gene exchange between populations. To summarize, $N$. gallinarum forms specific populations in various breeding grounds based on geographical isolation, and then spreads among different geographical populations through poultry trading. As a result, there is not only significant genetic differentiation but also certain genetic exchanges among $N$. gallinarum populations. The haplotypes of $N$. gallinarum based on mitochondrial tandem genes ( $\mathrm{COI}, \mathrm{COIl}$, and $\mathrm{NDS}$ ) show genetic variation, but haplotypes from the same geographical population do not follow certain rules with regard to their geographical areas, and the geographical genetic structure of $N$. gallinarum does not form significant genetic differences, according to the analysis of the haplotype phylogenetic tree and the haplotype network intermediate diagram. Multiple star-like distributions were found in the haplotype network intermediary maps based on tandem genes. Haplotypes from the same geographic population were not all clustered in the same clade, but were dispersed throughout each clade. All of the data based on the sequences of mitochondrial tandem genes (COI, COIl, and ND5), as well as the AMOVA analysis results among populations, show that the genetic differentiation of the $N$. gallinarum population was significant, and that genetic variation primarily comes from individuals within the population, but that genetic differentiation among populations was relatively small, indicating that the genetic differentiation of the $\mathrm{N}$. gallinarum population was relatively small. The neutral test and nucleotide pair difference analysis using three types of molecular markers revealed that $D$ of Tajima and $F s$ of Fu were both positive, with $P$ values that were not significant. 
The nucleotide pair difference maps of the entire population and each geographical population revealed a multi-peak distribution, indicating that the mite had not undergone any significant population expansion in the recent historical period and that the population size was relatively stable.

The expression of nucleotide polymorphism and the intermediate map of the haplotype network can also be used to determine population historical trends. If the haplotype network's middle graph shows a single star distribution, it means the population has experienced rapid re-expansion after the bottleneck [21]. Low haplotype diversity and low nucleotide diversity among nucleotide polymorphisms suggest $\left(\mathrm{H}_{d} \mathbb{\otimes} \mathrm{D} .5\right.$, piख0.005) that this population has recently experienced bottlenecks.

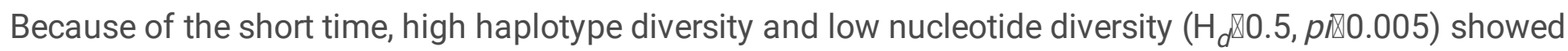
that, despite the population expanding quickly and recovering to the original population's number of individuals after the bottleneck effect, high genetic variation did not accumulate.

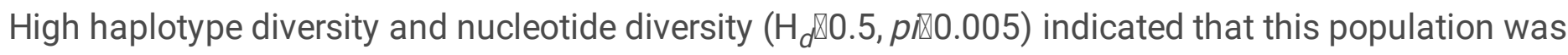
historically large and stable [22].The haplotypes in the intermediate map of the haplotype network constructed based on the tandem gene sequence are distributed in multiple stars in this paper, and the nucleotide polymorphisms of individual genes and tandem genes in different populations are characterized by high haplotype diversity and high nucleotide diversity, indicating that the population size of $N$. gallinarum has remained relatively stable in modern history. According to $N$. gallinarum epidemiological investigation and control research, the majority of $N$. gallinarum-infested farms are freerange households. The breeding grounds' playground is densely forested, with bamboo forests, grasslands, etc., providing ideal living conditions for the development and reproduction of $N$. gallinarum.

The larval stage of $N$. gallinarum parasitizes the surfaces of birds. They fall off the surface after being sucked to a certain extent and grow into adults in the grass and other vegetation, while the nymph lives on the plant juices. Farmers' traditional acaricides primarily target the surface or bedding of the poultry, ignoring the eggs and adults in the surrounding environment. Although acaricides can reduce $N$. gallinarum reproduction to some extent, different acaricides have different effects on $N$. gallinarum at different stages of development. No single acaricide can kill $N$. gallinarum eggs, larvae, and adults at the same time. These factors could explain why the population size of $N$. gallinarum has remained stable in recent years.

\section{Conclusion}

The $N$. gallinarum population showed high genetic diversity based on mitochondrial tandem genes analysis. Despite the fact that geographic isolation causes certain genetic differentiation among populations, $N$. gallinarum spread across different geographical populations as a result of human trade activities, and there was some gene exchange. It is clear that the $N$. gallinarum population is genetically distinct. The genetic variation is mostly found within the population, and there is no discernible genetic or 
geographical structure. Furthermore, the population size is relatively stable, and there has been no significant population growth in the recent historical period.

\section{Abbreviations}

COI:Cytochromecoxidase subunit l; COIl: Cytochromecoxidase subunit II; ND5: NADH dehydrogenase subunit 5; $F_{\mathrm{ST}}$ : Fixation index; $\mathrm{Nm}$ : Gene flow; PCR: Polymerase chain reaction.

\section{Declarations}

\section{Acknowledgments}

We thank the farm owners and staff for their assistance in sample collection during this study.

\section{Funding}

This work was supported in part by the Key Research and Development Program of Guangdong Province of China (2019B020218004) and the Project of Department of Education of Guangdong Province of China (2021KTSCX010).

\section{Availability of data and materials}

The dataset supporting the conclusions of this article is available in the GenBank repository under accession numbers OL587653هOL587674 (COI), OL906300खOL906312 (COI), OL906313هOL906329 $(N D 5)$.

\section{Authors'contributions}

All authors contributed to the collection of mosquitoes. $R L$ and $Y W$ conceived and designed the experiments. JT performed the experiments. YZ provided technical assistance. JT drafted the manuscript, and $S A$ revised the manuscript. All authors read and approved the final manuscript.

\section{Ethics approval and consent to participate}

This study was performed strictly according to the recommendations of the Guide for the Care and Use of Laboratory Animals of the Ministry of Health, China. Animals sampled were handled following established procedures of the Chinese Laboratory Animal Administration Act of 2017. The research protocol was reviewed and approved by the Research Ethics Committee of the South China Agricultural University.

\section{Consent for publication}

Not applicable. 


\section{Competing Interest}

The authors declare that they have no competing interests.

\section{References}

1. Sugimoto M. On the Formosan chicken mite, Neoschngastia gallinarum (Hatori, 1920). J Soi Trop Agr Formosa. 1936;8:241-253 (In Japanese).

2. Wang LM, Wang ZX, Weng YB, Lin RQ. The killing test In vitro of Chinese traditional medicine extracts and chemical drugs against Neoschngastia gallinarum. Poultry Husbandry and Dis Control. 2018;10:4142 (In Chinese).

3. Jeu MH. Experimental studies on the hibernation of chigger mites (Acariformes, Trombiculidea). (studies on Tsutsugamushi part XVIII). Acta Entomol Sinica. 1961;1:485-492 (In Chinese).

4. Zhou Q, Wang ZX, Tao JM, Qin JP, Lu JP, Lin RQ, Wang LM, Weng YB, Tan ZJ. Characterization of Neoschoengastia gallinarum from subtropical China by rDNA and identification of two genotypes based on mitochondrial cox1. Parasitol Res. 2020; 119: 3339-3345.

5. Poon RWS, Tam EWT, Lau SKP, Cheng VCC, Yuen K, Schuster RK, et al. Molecular identification of cestodes and nematodes by cox1 gene real-time PCR and sequencing. Diagn Micr Infec Dis. 2017;89:185190.

6. Shahbazi AE, Saidijam M, Maghsood AH, Matini M, Haghi MM, Fallah M. Genotyping of Fresh and Parafinized Human Hydatid Cysts Using nad1 and cox1 Genes in Hamadan Province, West of Iran. Iran J Parasitol. 2020;15:259-265.

7. Jørgensen TE, Karlsen BO, Emblem Å, Breines R, Andreassen M, Rounge TB, et al. Mitochondrial genome variation of Atlantic cod. BMC research notes. 2018;11:397.

8. Sriboonlert A, Wonnapinij P. Comparative mitochondrial genome analysis of the fi-refly, Inflata indica (Coleoptera: Lampyridae) and the first evidence of heteropl-asmy in fireflies. Int J Biol Macromol. 2019;121:671-676.

9. Simon C, Buckley TR, Frati F, Stewart JB, Beckenbach AT. Incorporating Molecular Evolution into Phylogenetic Analysis, and a New Compilation of Conserved Polymerase Chain Reaction Primers for Animal Mitochondrial DNA. Annual review of ecology, evolution, and systematics. 2006;37:545-579.

10. Tamura K, Peterson D, Peterson N, Stecher G, Nei M, Kumar S. MEGA5: molecular evolutionary genetics analysis using maximum likelihood, evolutionary distance, and maximum parsimony methods. Mol Biol Evol. 2011;28:2731-9. 
11. Zhang D, Gao F, Jakovlić I, Zou H, Zhang J, Li WX, et al. PhyloSuite: An- integrated and scalable desktop platform for streamlined molecular sequence data management and evolutionary phylogenetics studies. 2020;20:348-355.

12. Wilgenbusch JC, Swofford D. Inferring evolutionary trees with PAUP*. Current protocols in bioinformatics. 2003;Chapter 6:Unit 6.4.

13. Librado P, Rozas J. DnaSP v5: a software for comprehensive analysis of DNA pol-ymorphism data. Bioinformatics (Oxford, England). 2009;25:1451-2.

14. Excoffier L, Lischer HEL. Arlequin suite ver 3.5: a new series of programs to perform population genetics analyses under Linux and Windows. Mol Ecol Resour. 2010;10:564-7.

15. Wright S. The genetical structure of populations. Annals of eugenics. 1951;15:323-54.

16. Bandelt HJ, Forster P, Röhl A. Median-joining networks for inferring intraspecific phylogenies. Mol Biol Evol. 1999;16:37-48.

17. Chitimia L, Lin R, Cosoroaba I, Wu X, Song H, Yuan Z, et al. Genetic characteriz-ation of ticks from southwestern Romania by sequences of mitochondrial cox 1 and nad5 genes. Experimental \& applied acarology. 2010;52:305-11.

18. Gawryluk RMR, Gray MW. An ancient fission of mitochondrial cox1. Mol Phylo-genet Evol. 2010;27:710.

19. Paternina LE, Verbel VD, Bejarano EE. Comparison of $16 \mathrm{~S}$ and $\operatorname{cox} 1$ genes mitochondrial regions and their usefulness for genetic analysis of ticks (Acari: Ixodidae). Biomedica : revista del Instituto Nacional de Salud. 2016;36:295-302.

20. Ruiz EA, Rinehart JE, Hayes JL, Zúñiga G. Effect of geographic isolation on gen-etic differentiation in Dendroctonus pseudotsugae (Coleoptera: Curculion-idae). Hereditas. 2009;146:79-92.

21. So NKUL, Van HJKJ, Volckaert FAM. Genetic diversity and population history of the migratory catfishes Pangasianodon hypophthalmus and Pangasius bocourti in the Cambodian Mekong River. Fisheries Sci. 2006; 72:469-476.

22. Grant WS, Bowen BW. Shallow population histories in deep evolutionary lineages of marine fishes: insights from sardines and anchovies and lessons for conservat-ion. The Journal of heredity. 1998;89:415.

\section{Tables}

Table 1 Information about the populations of $N$. gallinarum used in this study 


\begin{tabular}{|lllll|}
\hline population cade & Collection site & Date & Coordinates & Sample size \\
\hline Guangdong (GD) & Foshan & July 2019 & $23^{\circ} 02^{\prime} \mathrm{N}, 113^{\circ} 06^{\prime} \mathrm{E}$ & 37 \\
& Yunfu & July 2019 & $22^{\circ} 93^{\prime} \mathrm{N}, 112^{\circ} 04^{\prime} \mathrm{E}$ & 31 \\
& Heshan & July 2019 & $22^{\circ} 76^{\prime} \mathrm{N}, 112^{\circ} 96^{\prime} \mathrm{E}$ & 33 \\
\hline Guangxi (GX) & Yulin & August 2019 & $22^{\circ} 19^{\prime} \mathrm{N}, 109^{\circ} 39^{\prime} \mathrm{E}$ & 27 \\
& Wuzhou & October 2019 & $23.29^{\prime} \mathrm{N}, 111.20^{\prime} \mathrm{E}$ & 14 \\
\hline Fujian (FJ) & Zhangzhou & October 2019 & $23^{\circ} 51^{\prime} \mathrm{N}, 117^{\circ} 66^{\prime} \mathrm{E}$ & 25 \\
\hline Jiangxi (JX) & Ji'an & May 2020 & $25^{\circ} 58^{\prime} \mathrm{N}, 113^{\circ} 46^{\prime} \mathrm{E}$ & 25 \\
\hline
\end{tabular}

Table 2 Haplotypes and nucleotide diversity of $N$. gallinarum population

\begin{tabular}{|lllllll|}
\hline population cade & sample size & $\mathrm{Mh}$ & $\mathrm{S}$ & $\mathrm{K}$ & $H_{d} \pm \mathrm{SD}$ & Pi $\pm \mathrm{SD}$ \\
\hline $\mathrm{GD}$ & 101 & 31 & 1309 & 668.957 & $0.930 \pm 0.012$ & $0.43886 \pm 0.00102$ \\
\hline GX & 41 & 27 & 828 & 376.474 & $0.978 \pm 0.011$ & $0.24257 \pm 0.00089$ \\
FJ & 25 & 4 & 42 & 13.760 & $0.860 \pm 0.017$ & $0.00718 \pm 0.00217$ \\
JX & 25 & 14 & 75 & 30.707 & $0.950 \pm 0.032$ & $0.01986 \pm 0.00112$ \\
\hline Total & 192 & 75 & 159 & 41.450 & $0.965 \pm 0.014$ & $0.26069 \pm 0.00109$ \\
\hline
\end{tabular}

Abbreviations: number of haplotypes $(H)$, number of segregating sites $(S)$, average number of nucleotide differences $(K)$, haplotype diversity $\left(H_{d}\right)$, and nucleotide diversity $(P I)$

*Population codes as in Table 1

Table 3 Pairwise genetic distance $\left(F_{S T}\right)$ and gene flow $\left(\mathrm{N}_{m}\right)$ of $N$. gallinarum population in China. Genetic distance $\left(F_{S T}\right)$ and gene flow $\left(\mathrm{N}_{m}\right)$ are shown below.

\begin{tabular}{|lllll|}
\hline Population code & GD & GX & FJ & JX \\
\hline GD & 0 & 2.34794 & 1.17037 & 0.34388 \\
GX & 0.09623 & 0 & 0.62541 & 0.53289 \\
\hline FJ & 0.17601 & 0.28558 & 0 & 0.20353 \\
JX & 0.42096 & 0.31933 & 0.55123 & 0 \\
\hline
\end{tabular}


Table 4 AMOVA analysis of concatenated mitochondrial genetic markers in N. gallinarum populations

\begin{tabular}{lcclll} 
Source of variation & d.f & Sum of & Variance & Percent of & Fixation Index \\
& & squares & components & variation & \\
\hline Among population & 3 & 687.905 & $6.73928 \mathrm{Va}$ & 27.06 & 0.27063 \\
\hline Within population & 188 & 2646.630 & $11.71225 \mathrm{Vb}$ & 72.94 & P-value $=0.00000$ \\
\hline Total & 191 & 3334.630 & 19.30202 & - &
\end{tabular}

Table 5 Tajima's $D$ and Fu's Fs tests for $N$. gallinarum geographic populations

\begin{tabular}{llllll} 
Population code & Sample size & Tajima's $D$ & $P$-value & Fu's $F s$ & $P$-value \\
\hline GD & 101 & 2.05291 & 0.74000 & 7.78941 & 0.96300 \\
\hline GX & 41 & 2.17554 & 0.98600 & 0.54408 & 0.63200 \\
\hline JX & 25 & 0.90502 & 0.86600 & 5.58319 & 0.97100 \\
\hline FJ & 25 & 2.13395 & 0.99600 & 4.54232 & 0.96100
\end{tabular}

\section{Figures}

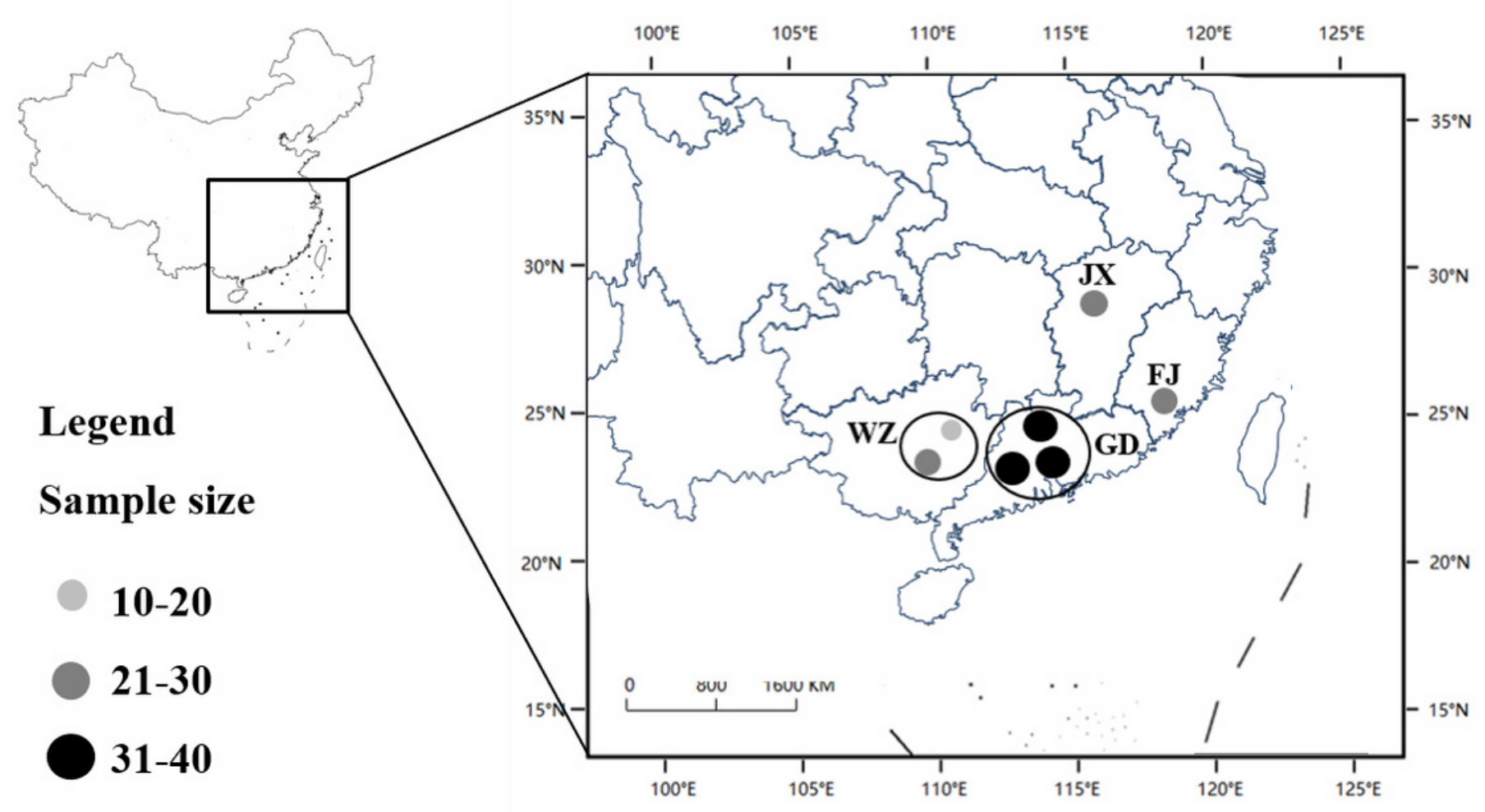


Figure 1

Schematic map of China showing sampling sites for $N$. gallinarum. The color of the circles indicates the sample size of the collecting localities, and the legend marked on the map (see Table 1 for abbreviations).

a COI

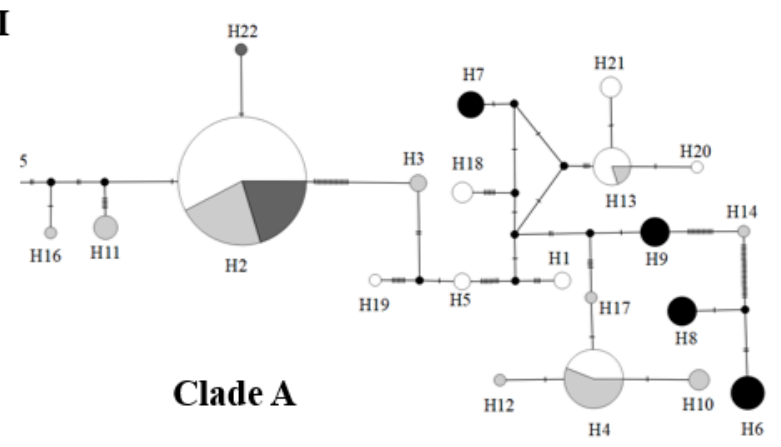

Clade B b COII

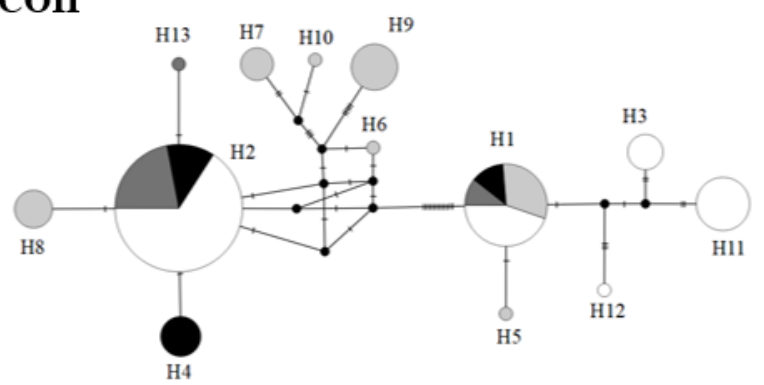

Clade A

\section{Clade B}

c ND5

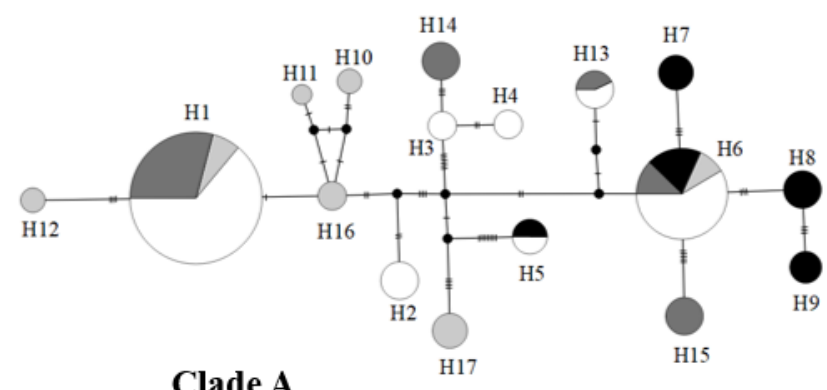

Clade A

Clade B
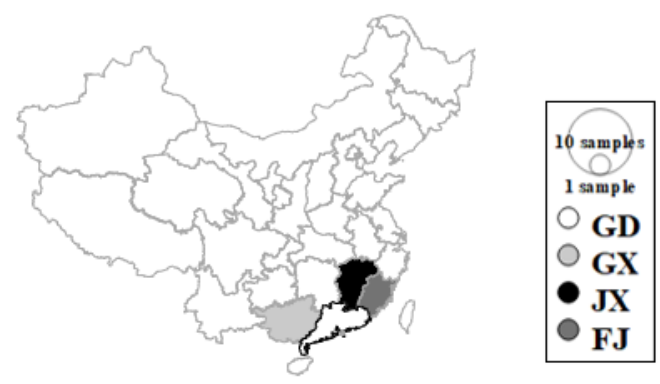

\section{Figure 2}

Median-joining haplotype network was generated using PopART 1.7 for the $N$. gallinarum.

Population corresponding to their geographical distribution separated into four populations in China. a Haplotype network of the $\mathrm{CO}$ gene. b Haplotype network of the COII gene. $\mathbf{c}$ Haplotype network of the ND5 gene. Colors represent different populations: GD = white; GX= light gray; JX = black, and FJ = charcoal grey. Each haplotype is represented by a circle in which the circle size is proportional to the haplotype frequency. Mutations between haplotypes are indicated by lines representing mutations from the common. 


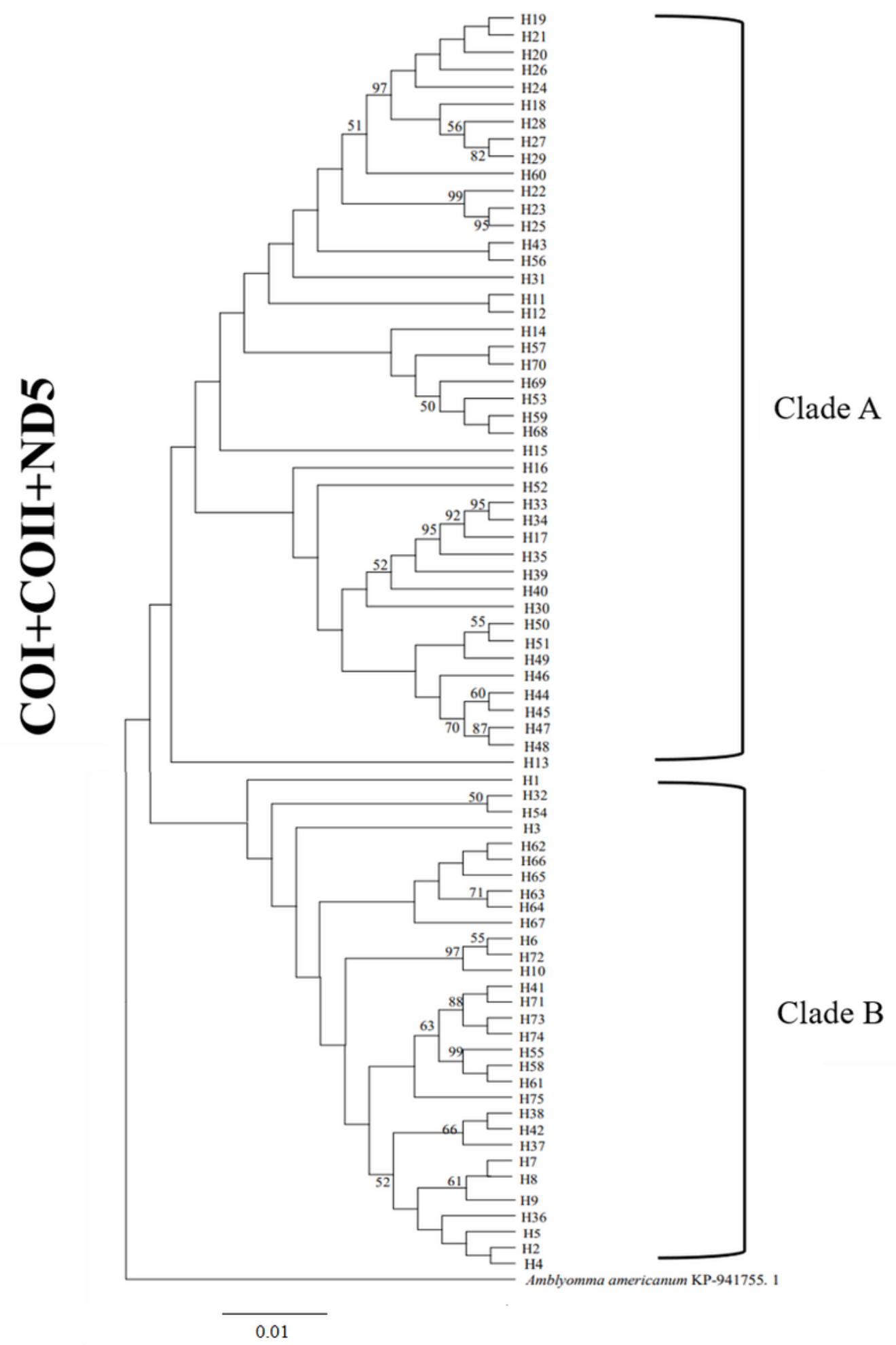

Figure 3

Phylogenetic relationships among 75 haplotypes of $N$. gallinarum. The phylogeny was inferred through maximum likelihood (ML) analysis of the concatenated nucleotide sequences based on substitution rates using the General Time Reversible model. 
Figure 4

Mismatch distribution of the N.gallinarum population. 